\title{
1 Csf1rb mutation uncouples two waves of microglia development in zebrafish
}

2

3

4

5

6

7

8

9

10

11

12

13

14

15

16 KEY WORDS: microglia, macrophages, zebrafish, csf1r, development, hematopoiesis

\author{
Giuliano Ferrero $^{1,2}$ ॠ, Magali Miserocchi ${ }^{1}, 2 \pi$, Elodie Di Ruggiero ${ }^{1}$ and Valérie \\ Wittamer ${ }^{1,2,3^{*}}$.
}

${ }^{1}$ Institut de Recherche Interdisciplinaire en Biologie Humaine et Moléculaire (IRIBHM), ${ }^{2}$ ULB Institute of Neuroscience (UNI), ${ }^{3}$ WELBIO, Université Libre de Bruxelles (ULB), Brussels, Belgium,

ף These authors contributed equally to this work

*Author for correspondence: Valerie Wittamer (valerie.wittamer@ulb.be)

Abstract word count: 178 words

Total text word count: 6237 words 


\section{ABSTRACT}

In vertebrates, the ontogeny of microglia, the resident macrophages of the 20 central nervous system, initiates early during development from primitive 21 macrophages. While murine embryonic microglia then persist through life, in zebrafish

22 these cells are transient, as they are fully replaced by an adult population originating 23 from larval hematopoietic stem cell (HSC)-derived progenitors. Colony-stimulating 24 factor receptor 1 (csf1r) is a fundamental regulator of microglia ontogeny in 25 vertebrates, including zebrafish which possess two paralogous genes: csf1ra and 26 csf1rb. While previous work showed invalidation of both genes completely abrogates 27 microglia development, the specific contribution of each paralog remains largely 28 unknown. Here, using a fate-mapping strategy to discriminate between the two 29 microglial waves, we uncover non-overlapping roles for csf1ra and csf1rb in 30 hematopoiesis, and identified csf1rb as an essential regulator of adult microglia development. Notably, we demonstrate that csf1rb positively regulates HSC-derived myelopoiesis, resulting in macrophage deficiency, including microglia, in adult mutant

33 animals. Overall, this study contributes to new insights into evolutionary aspects of 34 Csf1r signaling and provides an unprecedented framework for the functional 35 dissection of embryonic versus adult microglia in vivo. 


\section{INTRODUCTION}

Microglia are tissue-resident macrophages that play key immune and housekeeping roles in the central nervous system (CNS) (Prinz et al., 2019; Sierra et al., 2019). During development, microglia supports neurogenesis by releasing trophic factors (Tong and Vidyadaran, 2016), efficiently engulfing apoptotic neurons (Peri and Nusslein-Volhard, 2008) and pruning supernumerary synapses (Paolicelli et al., 2011). In the adult brain, microglia actively protrude branches to monitor the CNS microenvironment and interact with other cell types in order to maintain homeostasis (Davalos et al., 2005; Nimmerjahn et al., 2005). The indispensable role of microglia to foster CNS homeostasis becomes evident in human genetic conditions causing microglia deficits or dysfunctions (Li and Barres, 2018), which can result in severe pathologies such as Nasu-Hakola disease (Paloneva et al., 2002) and adult onset leukoencephalopathy with spheroids (Rademakers et al., 2011). Moreover, microglia are regarded as key mediators of the severe and prolonged inflammatory response triggered by CNS damage, which represents a major therapeutic hurdle in neurodegenerative disorders (Colonna and Butovsky, 2017).

During embryogenesis, microglia arise from yolk sac-derived primitive macrophages, which seed the developing neuroepithelium before the onset of neurogenesis (Alliot et al., 1999; Boche et al., 2012; Cuadros et al., 1993; Herbomel et al., 1999). Lineagetracing studies performed in the mouse model showed these early microglia are maintained throughout life (Ginhoux et al., 2010), although later hematopoietic waves might partially contribute to the adult microglia pool (De et al., 2018). Similar to the mouse, microglia ontogeny in zebrafish initiates from amoeboid-shaped primitive macrophages, which colonize the neural tissue starting at 35 hours post-fertilization (hpf) and then differentiate into branched microglia at around $60 \mathrm{hpf}$ (Herbomel et al., 2001). Unlike their mammalian counterparts however, embryo-derived microglia do not maintain in zebrafish, and the adult microglial network is established through a second wave of progenitors that seed the brain parenchyma later during development,

64 fully replacing the initial population by the end of the juvenile stage (Ferrero et al., 2018; Xu et al., 2015). Cell transplantation and fate mapping experiments identified embryonic hematopoietic stem cells (HSCs) arising from the hemogenic endothelium in the dorsal aorta (DA), as the source of adult microglia in this model (Ferrero et al., 
2018). Collectively, these findings have opened new avenues of research regarding possible functional differences between the two zebrafish microglial waves, as well as between mouse and zebrafish adult microglia, owing to their distinct cellular origins. Although zebrafish genetic models deficient for each microglia population would facilitate such comparative studies, little is known regarding the genetic regulation of adult microglia ontogeny, and so far no viable mutant resulting in the specific loss of adult microglia has been reported.

The tyrosine kinase colony-stimulating factor receptor 1 (csf1r), also known as M-csfr, is a fundamental regulator of mononuclear phagocyte homeostasis in vertebrates (Stanley and Chitu, 2014). It is predominantly expressed in macrophages and their precursors (regardless of their developmental origin), and exhibits pleiotropic effects including cell proliferation, differentiation and survival. Accordingly, CSF1R deficiency in human and mouse leads to a dramatic reduction in tissue macrophage development, including microglia (Erblich et al., 2011; Oosterhof et al., 2018; Rojo et al., 2019). Once established, microglia also rely on CSF1R signaling for their maintenance in the brain parenchyma and can be efficiently depleted in the mouse brain through pharmacological blockade of CSF1R (Elmore et al., 2014; Squarzoni et al., 2014). In humans, deficiencies in CSF1R signaling have been associated to neurodegenerative disorders, further highlighting the central role of CSF1R in microglia homeostasis (Oosterhof et al., 2019; Rademakers et al., 2011). In vivo, two non-homologous cytokines serve as ligands for CSF1R: Csf-1 and Interleukin 34 (II34) (Lin et al., 2008; Stanley, 1977). Both show distinct spatial and cellular distribution in the brain parenchyma (Cahoy et al., 2008; Zeisel, 2015) and elicit both overlapping and non-redundant biological responses in regional microglia (Easley-Neal et al., 2019; Greter et al., 2012; Kana et al., 2019; Wang et al., 2012).

As a result of a teleost-specific whole genome duplication, zebrafish possess two paralogs of the csf1r gene: csf1ra and csf1rb (Braasch et al., 2006). Fish deficient in both genes $\left(c s f 1 r^{D M}\right)$ lack microglia from the embryonic to the adult stages (Oosterhof et al., 2018), thus mimicking the Csf1r $r^{-}$mouse phenotype (Dai, 2002; Ginhoux et al., 2010). In contrast, individual mutants exhibit a less severe microglial phenotype, characterized by a transient loss of microglia in csf1 $\mathrm{ra}^{-/-}$zebrafish embryos and a moderate reduction of adult microglia in both csf1 $\mathrm{ra}^{-/}$and $c s f 1 \mathrm{rb}^{-/-}$single mutants (Oosterhof et al., 2018). Based on these phenotypes, it was suggested that both 
101 paralogs exhibit redundant functions. This prompted us to revisit the precise contribution of each paralog to microglia ontogeny, in light of the newly established model of microglia ontogeny in zebrafish where the two distinct primitive and definitive

104 microglia populations temporally overlap. We previously demonstrated that the $\mathrm{kdrl}:$ Cre model offers a powerful tool to discriminate primitive macrophage-derived embryonic microglia from HSC-derived adult microglia in vivo (Ferrero et al., 2018).

107 Exploiting this approach, we uncovered non-overlapping functions for csf1ra and csf1rb and identified csf1rb as a unique regulator of adult microglia development. In addition, we also demonstrated a specific contribution for the csf1rb paralogue to HSCderived myelopoiesis, consistent with the specific HSC origin of the adult microglial population.

\section{RESULTS}

114 Csf1rb localizes to definitive hematopoiesis and to embryonic microglia during 115 embryogenesis.

Previous studies have documented the restricted expression of csf1ra in neural 117 crest-derived cells, early macrophages and microglia during embryonic development 118 (Caetano-Lopes et al., 2020; Herbomel et al., 2001; Parichy DM, 2000) (Fig. 1A-E), 119 as well as its role in primitive myelopoiesis (Herbomel et al., 2001). However, although 120 csf1rb has been previously linked to microglia biology (Mazzolini et al., 2019; 121 Oosterhof et al., 2018), its expression has not been assessed in the context of 122 developmental hematopoiesis and little is known about its specific functions. Using 123 whole in situ hybridization (WISH), we found that csf1rb exhibits an expression profile 124 distinct from that of csf1ra during embryogenesis, with no expression in either neural 125 crest or primitive macrophages. Rather, csf1rb transcripts are first detected at around $12630 \mathrm{hpf}$ in the otic vesicle, as well as in a small number of hematopoietic cells in the 127 posterior blood island (PBI) (Fig. 1F). The latter is consistent with expression in 128 erythro-myeloid progenitors (EMPs), which are transiently found in the developing embryo. At $36 \mathrm{hpf}$, expression of csf1rb increases in the otic vesicle, and appears in 130 cells located along the dorsal aorta (DA), a site at the onset of hematopoietic stem cell 131 (HSC) formation (Fig. 1G). Over subsequent stages, expression in the otic vesicle 132 disappears but expands in the DA and at $48 \mathrm{hpf}$ onwards, csf1rb expression is 
133 observed in the caudal hematopoietic tissue $(\mathrm{CHT})$, region of definitive hematopoiesis

134 (Fig. 1H). At $72 \mathrm{hpf}$, the developing thymus also contains csf1rb-expressing cells,

135 reminiscent of HSC-derived lymphoid progenitor immigrants (Fig. 1I, J). Notably,

136 although csf1ra and csf1rb show distinct spatial expression patterns during

137 development, both transcripts overlap in microglia in the brain and retina starting at 72

138 hpf (Fig. 1E, J).

139 Because expression of csf1rb was mainly found in sites of definitive 140 hematopoiesis, we performed WISH in runx1 mutant embryos, which lack HSCs.

141 While expression in the otic vesicle and in the PBI at $30 \mathrm{hpf}$ is normal, we observed a 142 strong reduction of csf1rb transcripts in the DA, CHT and thymus of homozygous 143 embryos, thus identifying csf1rb-expressing cells in these anatomical locations as 144 HSC-dependent (Fig. 1K-O). As expected, microglial expression of csf1rb was not 145 affected in run $\times 1^{\text {null }}$ embryos, consistent with their ontogenic relationship with primitive 146 macrophages, which are runx1-independent (Ferrero et al., 2018) (Fig. 10). 147 Collectively, these results indicate that csf1ra and csf1rb paralogs have 148 nonoverlapping distribution during early development, except for microglia.

Csf1rb expression is restricted to hematopoietic progenitor cells and microglia among adult mononuclear phagocytes

We next assessed transcript expression of csf1r paralogs in mononuclear 153 phagocytes isolated from adult tissues. As a source for these studies, we used $154 \mathrm{Tg}($ mhc2dab:GFP; cd45:DsRed) double transgenic fish, as we previously 155 demonstrated that the mhc2dab:GFP; cd45:DsRed transgene combination enabled 156 the isolation by FACS of pure populations of tissue macrophages (Wittamer et al., 157 2011), including resident microglia (Ferrero et al., 2018). As shown in Fig. 2A, csf1ra 158 was highly expressed in adult microglia, as well as in mononuclear phagocytes 159 isolated from whole kidney marrow (WKM), spleen, liver and skin. In contrast to the 160 ubiquitous expression pattern of csf1ra, we found high levels of csf1rb transcripts in 161 microglial cells, very little expression in WKM macrophages and no expression in skin 162 and spleen macrophages. Analysis of a publicly available WKM single cell dataset 163 (Lareau et al., 2017) confirmed the lower expression of csf1rb versus csf1ra in 164 macrophages, but also revealed major differences, with csf1rb being found specifically 
enriched within hematopoietic progenitor cells (Fig. 2B-E). Together with our WISH analyses, these results suggest that csf1rb expression labels blood progenitors through life and identified microglia as a unique population of mononuclear phagocytes to display csf1rb expression outside the WKM.

\section{Different roles of csf1ra and csf1rb during embryonic microglia development}

To study csf1r function in vivo, we used two zebrafish mutant lines with no functional csf1ra or csf1rb paralog. The zebrafish panther line carries a point mutation in csf1ra, replacing a valine by a methionine in position 614 (Parichy DM, 2000). This change induces an impaired functioning of the kinase activity of the receptor, resulting in the disruption of internal cell signaling. This model has been previously used to demonstrate the contribution of csf1ra to microglia development (Herbomel et al., 2001; Oosterhof et al., 2018) and constitutes therefore a valuable tool for our investigations. The zebrafish line sa1503 harbors a splice site mutation in the csf1rb gene, leading to the inclusion of 86 nucleotides from intron 11 and a premature stop codon (Fig. S1A). This nonsense mutation results into the synthesis of a truncated protein that lacks the receptor kinase domain and is expected to be non-functional. The presence of the non-spliced transcript was confirmed through RT-PCR and sequencing analyses, thus validating csf1rb loss-of-function in the mutant (Fig. S1B, C). Homozygous csf1rb $b^{\text {sa1503 }}$ fish exhibit normal external morphology and behavior and, like the csf1ra-- mutant, survive to adulthood.

Previous studies indicated that csf1ra is not required for early myelopoiesis, as primitive macrophages develop normally in csf1 $\mathrm{ra}^{-/-}$embryos (Herbomel et al., 2001). Extending these analyses, we found no effect on the number of $m f a p 4^{+}$primitive macrophages in csf1rb mutants, as determined using whole-mount in situ hybridization (Fig. 3A,B). To study whether both paralogs were simultaneously required for primitive myelopoiesis, we intercrossed the two single mutant lines and derived $c s f 1 \mathrm{ra} / \mathrm{b}$ double mutant embryos (hereafter referred to as $c s f 1 r^{D M}$ ). As shown in Fig. 3A,B, the complete loss of csf1r had no consequence on primitive macrophage ontogeny, as the number of $\mathrm{mfap}^{+}$cells in double mutants were similar to that of wildtype and single homozygous mutant embryos. This is consistent with our recent findings using the macrophage mpeg1:EGFP reporter line (Kuil et al., 2020). We next 
197 investigated the requirement of the different csf1r paralogs for the establishment of 198 embryonic microglia, which differentiate in the brain parenchyma from primitive 199 macrophages starting at $60 \mathrm{hpf}$ (Ferrero et al., 2018; Herbomel et al., 2001). As 200 readout for microglia differentiation, we analyzed by WISH the expression of apoeb, a 201 microglia signature gene. Quantification of $a p o e b^{+}$cells present in the optic tectum at 20272 hpf showed the number of microglia was dramatically decreased in csf1ra-deficient 203 embryos ( $0.8 \pm 0.4$ cells) when compared to wild-type (20.8 \pm 1.4 cells), unaffected in 204 csf1rb-depleted embryos (19.1 \pm 1.3 cells) and similarly strongly reduced in $c s f 1 r^{D M}$ embryos (0.8 \pm 0.5 cells) (Fig. 3C,D). These results indicate that independently, csf1ra and not csf1rb, is important for establishing the first wave of microglia during zebrafish embryogenesis.

Because it was previously reported that csf1ra-- embryos exhibit a partial recovery of microglia cells at $6 \mathrm{dpf}$ (Herbomel et al., 2001), we next examined the status of microglia in the mutants later during development using WISH. While the numbers of apoeb+ cells were stable from 3 to $6 \mathrm{dpf}$ in wild-type and csf1rb-- embryos 212 (approximately 20 cells per optic tectum), we observed a gradual increase in the 213 number of microglia (from $0.7 \pm 0.4$ cells at $3 \mathrm{dpf}$ to $9.7 \pm 1.1$ cells at $6 \mathrm{dpf}$ ) in embryos 214 carrying the csf1ra ${ }^{-/-}$mutation (Fig. 3E,F). At $6 \mathrm{dpf}$ microglia cell numbers in $\mathrm{csf1ra}^{-/-}$ 215 embryos accounted for approximately $50 \%$ of total microglia cells found in sibling controls. Interestingly, at the same developmental stage, repopulation of the brain 217 parenchyma by microglia was not observed in double mutant embryos, which 218 remained devoid of apoeb-expressing cells. This observation indicated that recovery 219 of microglia in csf1ra-deficient embryos is mediated by csf1rb, which suggested there 220 may be a compensatory role for csf1rb in microglia development in the absence of 221 csf1ra. However, when we FACS-sorted mpeg1:EGFP+ cells from the heads of $6 \mathrm{dpf}$ 222 wild-type and csf1 ra ${ }^{-/}$embryos, we found no significant difference in expression of 223 csf1rb transcripts between both genotypes (Fig. S2). Taken together, these data 224 suggest that the partial recovery of embryonic microglia in csf1ra ${ }^{-/-}$embryos is csf1rb225 dependent but does not require a compensatory increase in csf1rb mRNA.

We investigated the source of the repopulating microglial cells in csf1ra227 deficient embryos. Indeed, microglia recovery in these embryos could result either 228 from a delay of differentiation of primitive macrophages or from the early and atypical 
contribution of HSCs, the precursors of adult microglia. We discriminated between these two possibilities by crossing the csf1ra mutant line to $\mathrm{Tg}(\mathrm{kdrl}: \mathrm{Cre}$; bactin2:IoxP-

231 Stop-loxP-DsRedexpress (also known as ßactin:Switch-DsRed); mpeg1:EGFP) triple 232 transgenics (Fig. 3G). As we previously showed, primitive macrophage-derived 233 embryonic microglia are $\mathrm{GFP}^{+}$, DsRed ${ }^{-}$in this setup (Fig. $3 \mathrm{H}$ ), while mononuclear 234 phagocytes originating from EMPs or HSCs are $\mathrm{GFP}^{+}$, DsRed ${ }^{+}$, owing to the 235 hemogenic nature of their precursors (Ferrero et al., 2018). Confocal microscopy analysis of live embryos revealed that $\mathrm{GFP}^{+}$microglia present at $6 \mathrm{dpf}$ in csf1radeficient embryos did not express the DsRed transgene, thus demonstrating their lineage relationship with primitive macrophages (Fig. 3I). These findings indicate that recovered microglial cells in the csf1ra mutant share the same cellular origin as their wild-type counterparts and point to a delay of primitive macrophage differentiation as the cause of the observed phenotype.

\section{csf1rb is a regulator of definitive microglia}

Like the single homozygous mutants, $c s f 1 r^{D M}$ are viable and fertile, allowing for investigations into the role of Csf1r signaling in the establishment of definitive microglia. As a way to discriminate between embryonic and adult microglia in our analyses, we relied again on mutant fish carrying the kdrl:Cre; ßactin:Switch-DsRed; mpeg1:EGFP triple transgene (Fig. 4A) and performed confocal analyses of brain sections immuno-stained for GFP and DsRed. In line with previous findings, the density of $\mathrm{GFP}^{+}$microglia cells in the brain parenchyma was decreased $\sim 60 \%$ in single $\mathrm{csf}_{\mathrm{ra}} \mathrm{r}^{--}$and $\mathrm{csf}_{1} \mathrm{rb}^{-/-}$mutant fish as compared to their wild-type siblings (Fig. C,D,N) and showed a dramatic reduction (90\%) in adult animals lacking both paralogs (Fig. 4E,N). However, analyzes for DsRed transgene expression to assess their primitive or definitive identity revealed striking microglial phenotypes (Fig. 4F-O). In wild-type fish, all $m p e g 1^{+}$microglia were DsRed $^{+}$, as expected from their known HSC origin (Fig. 4 F,J,O). Similarly, GFP ${ }^{+}$microglial cells from csf1ra- fish also co-expressed DsRed, indicating that adult microglia ontogeny still occurs in the absence of csf1ra (Fig. 4

$258 \mathrm{G}, \mathrm{K}, \mathrm{O})$. In contrast, the majority of the remaining $\mathrm{GFP}^{+}$cells in csf1 $\mathrm{rb}^{-/}$animals were 259 found to be DsRed', thus excluding them as microglia derived from the adult wave 260 (Fig.4 H,L,O). Based on the lack of DsRed expression, these mpeg1:EGFP ${ }^{+}$cells likely 
261 represent residual primitive microglia. This is further supported by observations that in $c s f 1 r^{D M}$ animals, which lack primitive microglia, the very few cells present in the brain parenchyma all expressed DsRed (Figure 4 I,M,O). Collectively, these data indicate that csf1rb, and not csf1ra, is essential for establishing the definitive wave of microglia in zebrafish.

To characterize the developmental dynamics leading to the observed

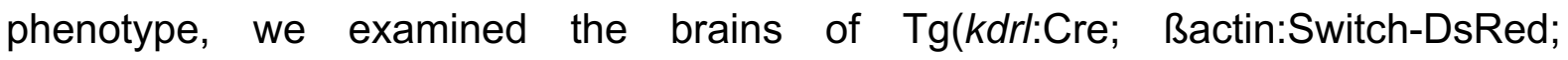
mpeg1:EGFP) wild-type and csf1ra or csf1rb mutants larvae at 21, 28, 35 and $50 \mathrm{dpf}$. As we previously reported, this time window encompasses the progressive replacement of $\mathrm{GFP}^{+}$DsRed ${ }^{-}$primitive microglia by definitive $\mathrm{GFP}^{+}$DsRed $^{+}$microglia in the brain parenchyma (Ferrero et al., 2018). These kinetic analyses revealed distinct phenotypes among the mutants. Consistent with our previous observations, in wild273 type fish the percentage of adult DsRed ${ }^{+}$microglia steadily increased over time (Fig. 274 4P). In contrast, the brain of csf1rb mutants remained largely devoid of DsRed ${ }^{+}$cells at all time points, suggesting that microglial progenitors fail to colonize the CNS in the absence of csf1rb (Fig. 4P). Surprisingly, in csf1ra ${ }^{\text {null }}$ animals we observed a shift in the emergence of adult microglia. At $21 \mathrm{dpf}$, when GFP ${ }^{+}$DsRed $^{-}$primitive microglia are still predominant in wild-type brains, the majority of microglia in csf1ra-/- fish already express DsRed (Fig. 4P). Based on these observations, we hypothesized that primitive microglia detected in the csf1ra ${ }^{-/}$brain at $6 \mathrm{dpf}$ fail to maintain through the juvenile stage. Interestingly, considering the overall density of $\mathrm{mpeg}^{+}$microglia across time, irrespective of the origin, we observed that in wild-type individuals the density of microglia increased from $1,7 \pm 0.07$ cells $/ \mathrm{mm}^{3}$ to $2,5 \pm 0.2$ cells $/ \mathrm{mm}^{3}$ between 21 and $50 \mathrm{dpf}$, mirroring the progressive expansion of the DsRed cells. In csf1rb mutants, microglia similarly expanded from $1,2 \pm 0.1$ to $2,2 \pm 0.3$ cells $/ \mathrm{mm}^{3}$ between 21 and $35 \mathrm{dpf}$ (Fig. 4Q). Given that these cells are from embryonic origin, such findings suggest that a partial compensation takes place in the brain of csf1rb-/ fish in the absence of DsRed ${ }^{+}$adult microglia. However, the potential of primitive microglia to compensate for the lack of the adult wave appears to be limited, since cell density dropped to $1,8 \pm 0.07$ cells $/ \mathrm{mm}^{3}$ at $50 \mathrm{dpf}$ (Fig. 4Q) and remained lower than

291 in wild-type fish throughout adulthood (Fig. 4N). The curve of microglia density across 292 time followed a different trend in csf1ra-/ fish, where DsRed ${ }^{+}$cells successfully 293 established in the brain by $21 \mathrm{dpf}$, but did not undergo the steady expansion that we 
observed in wild-type. This result suggests that, while dispensable for their ontogeny, csf1ra is likely required for maintaining adult microglia after they colonize the juvenile brain. Overall, we concluded that the csf1ra and csf1rb paralogs are respectively

297 required for the maintenance and specification of embryonic and adult microglia in 298 zebrafish and that individual loss of function of either paralogue results in reduced 299 microglia densities in the adult.

\section{csf1rb is required for the development of HSCs-derived myeloid cells}

We sought to dissect the mechanisms linking csf1rb to adult microglia development. Based on the expression profile of csf1rb in hematopoietic progenitors and the developmental relationship between adult microglia and HSCs, we hypothesized that csf1rb regulates definitive hematopoiesis in zebrafish. By WISH, we did not detect any significant alteration in the expression of runx1 in the DA of csf1rbdeficient embryos, indicating normal specification of the hemogenic endothelium (Fig. $5 \mathrm{~A}, \mathrm{~B})$. In addition, at 3 and $6 \mathrm{dpf}, c-m y b$ expression in the $\mathrm{CHT}$ and the pronephros, which specifically labels HSCs and progenitors, was not changed in csf1 rb-/ embryos (Fig. 5C-F). This demonstrates that neither the emergence of HSCs nor the maintenance of progenitors during embryonic development requires csf1rb.

We evaluated a possible requirement for csf1rb during HSC differentiation. In 313 the zebrafish embryo, T lymphopoiesis starts at around $50 \mathrm{hpf}$, with HSC-derived 314 thymocyte precursors migrating to the developing thymus (Hess and Boehm, 2012; 315 Murayama et al., 2006). We found that T cell development was not affected in the 316 absence of csf1rb, as expression of the early $\mathrm{T}$ cell marker rag1 was detected in 317 mutant embryos at levels similar to that seen in wild-type (Fig. 5G, H). Next, we 318 assessed the myeloid potential of csf1rb-deficient HSCs. As the different waves of 319 myelopoiesis temporally overlap during embryonic development, we used triple 320 transgenic kdrl:Cre; ßactin:Switch-DsRed; mpeg1:EGFP embryos to discriminate in 321 the CHT between newly born definitive macrophages (GFP ${ }^{+}$DsRed $^{+}$) and primitive macrophages (GFP ${ }^{+}$DsRed $)$having colonized the site from the periphery (Fig. $5 \mathrm{I}$ ). In 323 wild-type embryos, we found that for the first 48 hours of development, all GFP ${ }^{+}$ 324 macrophages present in the CHT (40 cells on average) are derived from primitive 325 hematopoiesis, as indicated by their lack of DsRed expression (Fig. 5J, K). The first 
definitive macrophages, identified as $\mathrm{DsRed}^{+} \mathrm{GFP}^{+}$cells, were detected in the CHT at around $48 \mathrm{hpf}$ ( $\sim 3$ cells on average) (Fig. $5 \mathrm{~J}, \mathrm{~K}$ ). This population then slowly increased over time, and at $6 \mathrm{dpf}$, the $\mathrm{CHT}$ contained on average 113 double positive cells per embryo. By that stage, definitive macrophages in the CHT outnumbered primitive macrophages, accounting for up to $80 \%$ of the total $\mathrm{GFP}^{+}$population. Having delineate the kinetics of differentiation of definitive macrophages in the $\mathrm{CHT}$, we next performed similar quantification of macrophage numbers in single mutants. In csf1ra-deficient embryos, we found that both the kinetics of appearance and total number of DsRed ${ }^{+}$ 334 GFP $^{+}$double positive cells were similar to wild-types, thus indicating that the developmental program of HSC-derived macrophages was not affected (Fig. 5K-O). In contrast, $c s f 1 \mathrm{rb}^{-/}$embryos exhibited significantly decreased numbers of definitive macrophages at each time points (4 versus 19 at $72 \mathrm{hpf}$ and 19 versus 113 at $6 \mathrm{dpf}$ ) (Fig. 5K-O). These findings demonstrate that Csf1rb activity specifically supports the embryonic development of HSC-derived macrophages. Interestingly, analysis of CHT $\mathrm{GFP}^{+}$primitive macrophages during the $48 \mathrm{hpf}$ to $6 \mathrm{dpf}$ time-window also revealed major phenotypic differences among the mutants. Consistent with previous observations (Herbomel et al., 2001) and our own results suggesting a role for csf1ra in controlling early macrophage invasion in embryonic tissues, we found that the CHT of csf1ra mutants became colonized by $30 \%$ less primitive macrophages (Fig. 5J). By comparison, the numbers of primitive macrophages present in the $\mathrm{CHT}$ of csf1rb mutant embryos were similar to that of wild-types. These findings suggest that in primitive macrophages the csf1rb paralogue is dispensable for cell migration. Collectively, these results demonstrate that csf1rb, and not csf1ra, is required for definitive myelopoiesis in the zebrafish embryo.

To evaluate whether Csf1rb functions are required for life, we examined WKM cell suspensions from mpeg1:EGFP transgenics by flow cytometry. These analyses revealed the relative percentage of mpeg1:EGFPhigh cells, which identify macrophages

353 in adult fish (Ferrero et al., 2020), was reduced from $1 \pm 0.2 \%$ for wild-type to $0.4 \pm$

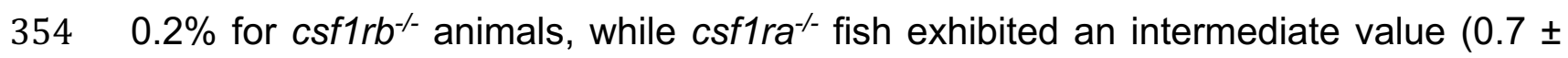
$3550.1 \%$ ) (Fig. 6A). Intriguingly, the mpeg1:EGFPlow population, which labels most $\lg ^{+}$ 356 B lymphocytes (Ferrero et al., 2020) was also barely detectable in csf1rb ${ }^{-/}$animals 357 (Fig. 6B) (Mean \pm SEM; wild-type: $13 \pm 3.6 \%$; csf1ra ${ }^{--}: 10.9 \pm 0.3 \%$; csf1rb-: $1.6 \pm$ 358 0.5\%). Csf1rb mutants also displayed a light-scatter profile distinct from that of wild- 
type and csf1ra ${ }^{-/}$fish, with a significant loss of the forward scatter (FSC) ${ }^{\text {hi }}$ side scatter $(\mathrm{SSC})$ hi myeloid gate (Mean \pm SEM, n=3; wild-type: $27.5 \pm 1.7 \%$; csf1ra--: $26 \pm 4.2 \%$; csf1rb $^{-/-}: 16.3 \pm 1.7 \%$ ), as well as an increase of the $\mathrm{FSC}^{\text {hi }} \mathrm{SSC}^{\text {lo }}$ progenitor fraction (Mean \pm SEM; wild-type: $22.2 \pm 1.8 \%$; csf1ra ${ }^{-/}: 28.6 \pm 5.8 \%$; csf1rb-/: $45.5 \pm 1.6 \%$ ) (Fig. 6B). As the myeloid fraction mostly contains mature neutrophils, these observations suggest a complete block at the myeloid progenitor stage. Finally, and in line with the change in mpeg1-expressing $B$ lymphocytes, the relative percentage of $\mathrm{FSC}^{\text {loSSClo }}{ }^{1}$ lymphoid cells was also impaired in $\mathrm{csf1rb}^{-/}$animals (Mean $\pm \mathrm{SEM}$; wild-type: $36.2 \pm 2.2 \%$; csf1ra ${ }^{--:} 34.3 \pm 3.6 \%$; csf1rb ${ }^{-/}: 21.7 \pm 0.4 \%$ ) (Fig. 6A). Collectively, these findings indicate the absence of csf1rb results in functional deficiencies in myelopoiesis in the adult, and a concomitant lack of mpeg1-expressing B lymphocytes.

\section{DISCUSSION}

Tissue macrophages constitute a highly heterogeneous compartment, based on their origin and the niche they inhabit (Bennett and Bennett, 2019; Guilliams et al., 2020). CSF1R signalling is a common pathway regulating the development of most macrophages in vertebrates, as demonstrated by their dramatic loss (including microglia) in Csf1r-deficient mice and csf1r-deficient zebrafish (Dai, 2002; Kuil et al., 2020; Oosterhof et al., 2018; Rojo et al., 2019). However, while CSF1R is represented only once in mammalian genomes, zebrafish possess two copies of the gene and their relative contribution to myelopoiesis has remained unknown. Focusing on the specific context of microglia ontogeny, in this study we have thus investigated the functions of each paralog during macrophage development. While our gene expression analyses demonstrate that csf1rb, like csf1ra, is expressed within the hematopoietic compartment, they also reveal a divergence in their expression profiles, with csf1ra expressed in all tissue macrophages (regardless of their primitive or definitive origin) and csf1rb restricted to microglia and definitive blood progenitors. Given that in the mouse Csf1r is expressed all throughout the path of macrophage differentiation (from hematopoietic progenitors to mature cells) (Hawley et al., 2018; Sasmono et al., 2003), such complementary patterns suggest that subfunctionalization, a process where the two gene copies partition the ancestral function (Force et al., 1999), may have 
contributed to the evolution of this family in zebrafish. Accordingly, csf1ra signalling is required for the establishment of primitive macrophage-derived embryonic microglia

393 (Herbomel et al., 2001; Oosterhof et al., 2018), while csf1rb controls the ontogeny of 394 definitive macrophages, including adult microglia. Our work thus demonstrates that csf1ra and csf1rb are jointly required to fulfil the roles of mammalian CSF1R in myelopoiesis.

Interestingly, despite these functional divergences, we also provide evidence that csf1rb is able to compensate, at least partially, for the absence of csf1ra. For example, whereas csf1rb loss has no effect on microglia development in embryos with a functional csf1ra paralog, csf1rb signalling is responsible for the partial recovery of microglia observed in csf1ra ${ }^{-/}$larvae, as indicated by the absence of recovery in $c s f 1 r^{D M}$ mutants. Since microglia repopulating $c s f 1 \mathrm{ra}^{-/-}$larvae entirely derived from primitive macrophages and did not exhibit a compensatory overexpression of csf1rb, it thus appears that in this setting the basal expression level of csf1rb on embryonic microglia is sufficient for driving the recovery process. Our findings also provide new insights into the functions and complex interplay between csf1r paralogs and the three Csf1r ligands identified in zebrafish: Interleukin-34 (II34), Csf1a and Csf1b. Similar to the mouse (Greter et al., 2012; Wang et al., 2012), Interleukin-34 acting through Csf1ra is thought to control the migration of primitive macrophages to the embryonic neuroepithelium in zebrafish (Kuil et al., 2019; Wu et al., 2018). Accordingly, il34deficient embryos phenocopy both the microglial loss at $3 \mathrm{dpf}$ and the partial recovery at $5 \mathrm{dpf}$ observed in csf1ra mutants (Herbomel et al., 2001; Kuil et al., 2019). Given that $c s f 1 r^{D M}$ larvae are completely devoid of microglia at $6 \mathrm{dpf}$, this suggests that csf1rb-mediated microglia replenishment in csf1ra ${ }^{-/}$larvae is independent of II34 signalling. Also, since the very few microglia present at $3 \mathrm{dpf}$ in csf1ra or il34-deficient embryos retain their proliferative capacity and can be increased by csf1a overexpression (Kuil et al., 2019; Oosterhof et al., 2018), it is tempting to speculate that csf1a and/or csf1b signalling through csf1rb triggers the proliferation of embryonic microglia in csf1ra mutants. In line with this interpretation, we recently showed that primitive macrophages in $c s f 1 r^{D M}$ embryos, which lose interaction with all Csf1r 421 ligands, exhibit both migration and proliferation defects (Kuil et al., 2020). Further investigation in zebrafish mutants combining Csf1 ligands and receptors knockout will be instrumental in testing this hypothesis. 
A major finding of our study is the demonstration that the second microglial wave in zebrafish is completely abolished in absence of csf1rb, thus uncovering a selective role for csf1rb in the establishment of HSC-derived adult microglia. Indeed, although microglia cells are present in each single mutant (albeit at comparably reduced cell density), lineage tracing of definitive microglia development revealed the microglial population presents in the brain of $c s f 1 \mathrm{rb}^{-/}$adult fish remain of primitive 430 origin. This is in sharp contrast to csf1ra-deficient and wild-type fish, where the HSCderived adult population fully replaces the primitive microglial pool. Interestingly, however, although adult microglia develop normally in csf1ra-deficient juvenile fish, 433 their numbers drop towards the adult stage. This suggests that while being 434 dispensable for the ontogeny of the definitive microglial wave, csf1ra likely contributes to microglia maintenance within the adult brain parenchyma. The opposite microglial phenotypes observed in csf1ra and csf1rb mutants also shed light on potential dynamics between the two microglial waves during development. Through timecourse analyses, we found that the incomplete recovery of primitive microglia in csf1ra juveniles is compensated by an earlier establishment of the definitive wave compared to wild-type. Conversely, the lack of definitive microglia in csf1rb mutants results in the primitive pool being retained in the adult. By analogy with the current view established in the mouse model (Guilliams et al., 2020), it is conceivable that competition for the juvenile brain niche regulates the exchange between the two microglial waves, in a scenario where efficient seeding of the brain by definitive microglia would require the regression of the primitive wave. Another plausible hypothesis is that the adult wave may actively participate to the removal of the primitive population, therefore explaining the maintenance of embryonic microglia in csf1rb-deficient adult animals. Future studies, making use of new and more sophisticated tools, will be required to address these complex questions. Nevertheless, since neither definitive microglia in csf1ra mutants nor primitive microglia in csf1rb mutants achieved the cellular density seen in wild-type adults, other intrinsic or extrinsic factors aside from niche availability are likely to affect microglia homeostasis in the adult brain.

Our investigations into the molecular mechanisms underlying the microglial phenotype of csf1rb mutant fish revealed that HSCs give rise to myeloid cells in a 
macrophages during embryonic development and in the adult hematopoietic niche.

458 These findings are consistent with the runx1-dependent selective expression of csf1rb on blood progenitor cells throughout life. In addition, csf1rb is dispensable for HSC emergence in the AGM and, unlike csf1ra, does not seem to control cell migration to the different niches. On the whole, this suggests that the depletion of adult microglia and definitive macrophages in the csf1rb mutant results from a deficit of differentiation at the level of HSC-derived myeloid progenitors. These data add to previous findings in zebrafish (Yu et al., 2017) and mouse (Azzoni et al., 2018) showing that distinct molecular mechanisms regulate the emergence of subsequent macrophages waves. Overall, zebrafish csf1ra and csf1rb mutants may thus provide insightful models for the functional dissection of each microglial population and to better understand microglia development from an evolutionary perspective.

Finally, a surprising finding of our study is that fish lacking the csf1rb paralog also lack a population of $m p e g 1^{+} \mathrm{B}$ cells in the WKM. As we previously showed that these cells account for the majority of IgM-expressing B lymphocytes in zebrafish 472 (Ferrero et al., 2020), these observations suggest that B lymphopoiesis is globally 473 impaired in csf1rb mutant animals. This is interesting because while no such phenotype has been reported in adult CSF1R-deficient mice so far, expression of CSF1R was recently identified on a subset of embryonic myeloid-primed B-cell progenitors in the fetal liver, and its loss associated to defective fetal B-cell differentiation in vivo (Zriwil et al., 2016). Although the precise contribution of csf1rb to zebrafish B lymphopoiesis remains to be investigated, our work thus provides further support for a role of CSF1R beyond myelopoiesis in vertebrates. Also, because in the 480 mouse the fetal wave of B lymphopoiesis mainly produces innate-like B-1 lymphocytes, and given the B-cell phenotype similarities between Csf1r-deficient mice and $c s f 1 r^{-/-}$zebrafish, this study also adds to the growing view that mammalian B-1 lymphocytes and teleost adult B cells could be evolutionary related (Scapigliati et al., 2018). 


\section{MATERIALS AND METHODS}

489

490

491

492

493

494

495

496

497

498

499

500

501

502

503

504

505

506

507

508

509

510

511

512

513

514 Whole Mount In Situ Hybridization (WISH)

\section{Zebrafish husbandry} CGCTCCTGCTCCAAGAACCTG-3').

\section{Flow cytometry and cell sorting}

\section{Quantitative PCR}

Zebrafish were maintained under standard conditions, according to FELASA guidelines (Alestrom et al., 2019). All experimental procedures were approved by the ethical committee for animal welfare (CEBEA) from the ULB. The following lines were used: Tg(mpeg1:EGFP)gl22 (Ellett et al., 2011); Tg(kdrl:Cre) $)^{s 89}$ (Bertrand et al., 2010); Tg(actb2:IoxP-STOP-IoxP-DsRed $\left.{ }^{\text {express }}\right)^{\text {sd5 }}$ (Bertrand et al., 2010); panther ${ }^{\text {je1 }}$ (Parichy DM, 2000); csf1rbsa1503 mutants were generated via ethyl-nitrosurea (ENU) mutagenesis by the Sanger Institute Zebrafish Mutation Project. The following primers were used to identify the point mutation in intron 11 by PCR on genomic DNA: sa1503F (5'-CTCTCTCTGTGGCAACTCTATGGATG-3'); sa1503R

Single-cell suspensions of zebrafish whole embryos or adult WKM were prepared as previously described (Ferrero et al., 2018). Heads of $6 \mathrm{dpf}$ zebrafish larvae were rapidly dissected in ice-cold PBS and then processed as the other samples. Flow cytometry and cell sorting were performed with a FACS ARIA II (Becton Dickinson). Analyses were performed using the FlowJo software (Treestar).

RNA extraction from sorted cells and cDNA synthesis were performed as described (Ferrero et al., 2020). Biological triplicates were compared for each subset. Relative amount of each transcript was quantified via the $\Delta$ Ct method, using elongation-Factor1-alpha (ef1 $\alpha$ ) expression for normalization. Primers used are reported in Table 1. 
515 Probes for apoeb, runx, c-myb, mfap4 and csf1rb were synthesized in-vitro. For the

516 csf1rb WISH, we combined two probes hybridizing to different portion of the transcript

517 to increase the signal strength. The following primers were used for the generation of

518 the csf1rb probes from zebrafish 4 dpf larvae cDNA: Fw1: 5'-

519 ATCATTGCAGTGCTGACCTGTATG; Rv1: 5'-

520 GGTGAGCTCCAGGTGAAGTTGTAG; Fw2: 5-'ATGGCCAACCAATCCATTTCTGAG

521 Rv2: 5'AGTAAGCATTCCTTGCGGGATGTT. Embryos or larvae were fixed in 4\%

$522 \mathrm{PFA}$ at $4^{\circ} \mathrm{C} \mathrm{O} / \mathrm{N}$ and then stored in methanol at $-20^{\circ} \mathrm{C}$. Whole-mount in-situ

523 hybridization was performed according to previously published protocols (Thisse and

524 Thisse, 2008).

525

\section{Immunofluorescence, tissue clearing and imaging}

527 Larvae were fixed in $4 \%$ PFA $\mathrm{O} / \mathrm{N}$ at $4^{\circ} \mathrm{C}$ and stored in methanol at $-20^{\circ} \mathrm{C}$. Adult 528 zebrafish brains were fixed 4 hours in $4 \%$ PFA at $4^{\circ} \mathrm{C}$, incubated in $30 \%$ sucrose/PBS 529 overnight and embedded in OCT (Leica) for cryosectioning. Immunofluorescence on 530 whole embryos or $30 \mu \mathrm{m}$-thick brain slices was performed as described (Ferrero et al., 531 2018), using chicken anti-GFP (1:500, Abcam), polyclonal rabbit anti-DsRed (1:500, 532 Takara) primary antibodies and goat-anti chicken Alexa 488 (1:500, Abcam), donkey 533 anti-rabbit alexa 594 (1:500, Abcam) secondary antibodies. Dissected brains from 534 larval or juvenile fish (21 to $50 \mathrm{dpf}$ ) were fixed in 4\% PFA at $\mathrm{pH} 8.5$ to preserve 535 endogenous fluorescence, and subsequently tissue-cleared using the CUBIC protocol 536 (Susaki et al., 2015), as described (Ferrero et al., 2018). Imaging was performed on a 537 Zeiss LSM 780 inverted microscope, using a Plan Apochromat 20x objective for adult 538 sections and a LD LCI Plan Apochromat 25x water-immersion objective for whole539 mount embryos and tissue-cleared brains. Images of entire adult brain sections were 540 obtained by combining 15 tiles, for a total area of $1.80 \mathrm{~mm}^{2}$. 


\section{ACKNOWLEDGEMENTS}

545 We thank Mireia Rovira, member of the Wittamer lab, for critical discussion and

546 comments on the manuscript. We are also grateful to Marianne Caron for technical

547 assistance and to Christine Dubois for help with flow cytometry.

548

\section{COMPETING INTERESTS}

550 The authors declare no competing financial interests.

551

\section{FUNDING}

553 This work was funded in part by a WELBIO Grant (WELBIO-CR-2015S-04), the Funds

554 for Scientific Research (FNRS) under Grant Numbers F451218F, UN06119F and

555 UG03019F, and the Minerve Foundation (to V.W.). G.F. is supported by a Research

556 Fellowship (FNRS), M.M. by a fellowship from The Belgian Kid's Fund and E.D. by a 557 fellowship from the Fund for Research Training in Industry and Agriculture (FRIA).

558

559 DATA AVAILABILTY

560 All datasets generated for this study are included in the manuscript/ Supplementary 561 Files.

562

\section{AUTHORSHIP CONTRIBUTION}

564 V.W. designed the research and directed the study. G.F., M.M and E.D. performed 565 experiments. G.F. and V.W. wrote the manuscript with comments from all authors. 


\section{REFERENCES}

568 Alestrom, P., D'Angelo, L., Midtlyng, P. J., Schorderet, D. F., Schulte-Merker, S., 569 Sohm, F. and Warner, S. (2019). Zebrafish: Housing and husbandry 570 recommendations. Lab Anim, 23677219869037.

571 Alliot, F., Godin, I. and Pessac, B. (1999). Microglia derive from progenitors, 572 originating from the yolk sac, and which proliferate in the brain. Brain Res Dev Brain 573 Res 117, 145-152.

574

575

576

Azzoni, E., Frontera, V., McGrath, K. E., Harman, J., Carrelha, J., Nerlov, C., Palis, J., Jacobsen, S. E. W. and de Bruijn, M. F. (2018). Kit ligand has a critical role in mouse yolk sac and aorta-gonad-mesonephros hematopoiesis. EMBO Rep.

Bennett, M. L. and Bennett, F. C. (2019). The influence of environment and origin on brain resident macrophages and implications for therapy. Nature Neuroscience.

Bertrand, J. Y., Chi, N. C., Santoso, B., Teng, S., Stainier, D. Y. and Traver, D. (2010). Haematopoietic stem cells derive directly from aortic endothelium during development. Nature 464, 108-111.

Boche, D., Perry, V. H. and Nicoll, J. A. (2012). Review: activation patterns of microglia and their identification in the human brain. Neuropathol Appl Neurobiol 39, 3-18.

Braasch, I., Salzburger, W. and Meyer, A. (2006). Asymmetric evolution in two fishspecifically duplicated receptor tyrosine kinase paralogons involved in teleost coloration. Mol Biol Evol 23, 1192-1202.

Caetano-Lopes, J., Henke, K., Urso, K., Duryea, J., Charles, J. F., Warman, M. L. and Harris, M. P. (2020). Unique and non-redundant function of csf1r paralogues in regulation and evolution of post-embryonic development of the zebrafish. Development 147.

Cahoy, J. D., Emery, B., Kaushal, A., Foo, L. C., Zamanian, J. L., Christopherson, K. S., Xing, Y., Lubischer, J. L., Krieg, P. A., Krupenko, S. A., et al. (2008). A transcriptome database for astrocytes, neurons, and oligodendrocytes: a new resource for understanding brain development and function. J Neurosci 28, 264-278.

Colonna, M. and Butovsky, O. (2017). Microglia Function in the Central Nervous System During Health and Neurodegeneration. Annu Rev Immunol 35, 441-468. 

the avian central nervous system. J Comp Neurol 330, 113-129. stimulating factor 1 receptor gene results in osteopetrosis, mononuclear phagocyte deficiency, increased primitive progenitor cell frequencies, and reproductive defects. Blood 99, 10. R., Dustin, M. L. and Gan, W. B. (2005). ATP mediates rapid microglial response to local brain injury in vivo. Nat Neurosci 8, 752-758. M. R. (2018). Two distinct ontogenies confer heterogeneity to mouse brain microglia. Development 145. CSF1R Ligands IL-34 and CSF1 Are Differentially Required for Microglia Development and Maintenance in White and Gray Matter Brain Regions. Front Immunol 10, 2199. mpeg1 promoter transgenes direct macrophage-lineage expression in zebrafish. Blood 117, e49-56. R. A., Kitazawa, M., Matusow, B., Nguyen, H., West, B. L., et al. (2014). Colonystimulating factor 1 receptor signaling is necessary for microglia viability, unmasking a microglia progenitor cell in the adult brain. Neuron 82, 380-397. of colony stimulation factor-1 receptor results in loss of microglia, disrupted brain development and olfactory deficits. PLoS One 6, e26317.

625 Ferrero, G., Gomez, E., Lyer, S., Rovira, M., Miserocchi, M., Langenau, D. M., 626 Bertrand, J. Y. and Wittamer, V. (2020). The macrophage-expressed gene (mpeg) 6271 identifies a subpopulation of B cells in the adult zebrafish. J Leukoc Biol 107, 431628443.

629 Ferrero, G., Mahony, C. B., Dupuis, E., Yvernogeau, L., Di Ruggiero, E., 630 Miserocchi, M., Caron, M., Robin, C., Traver, D., Bertrand, J. Y., et al. (2018). 
631 Embryonic Microglia Derive from Primitive Macrophages and Are Replaced by cmyb-

632 Dependent Definitive Microglia in Zebrafish. Cell Rep 24, 130-141.

633 Force, A., Lynch, M., Pickett, B. F., Amores, A., Yan, Y. and Postlethwait, J. 634 (1999). Preservation of Duplicate Genes by Complementary, Degenerative Mutations. 635 Genetics 151, 15.

636 Ginhoux, F., Greter, M., Leboeuf, M., Nandi, S., See, P., Gokhan, S., Mehler, M. 637 F., Conway, S. J., Ng, L. G., Stanley, E. R., et al. (2010). Fate mapping analysis 638 reveals that adult microglia derive from primitive macrophages. Science 330, 841-845.

639 Greter, M., Lelios, I., Pelczar, P., Hoeffel, G., Price, J., Leboeuf, M., Kundig, T. M., 640 Frei, K., Ginhoux, F., Merad, M., et al. (2012). Stroma-derived interleukin-34 controls 641 the development and maintenance of langerhans cells and the maintenance of 642 microglia. Immunity 37, 1050-1060.

643 Guilliams, M., Thierry, G. R., Bonnardel, J. and Bajenoff, M. (2020). Establishment 644 and Maintenance of the Macrophage Niche. Immunity 52, 434-451.

645 Hawley, C. A., Rojo, R., Raper, A., Sauter, K. A., Lisowski, Z. M., Grabert, K., Bain, 646 C. C., Davis, G. M., Louwe, P. A., Ostrowski, M. C., et al. (2018). Csf1r-mApple 647 Transgene Expression and Ligand Binding In Vivo Reveal Dynamics of CSF1R 648 Expression within the Mononuclear Phagocyte System. J Immunol 200, 2209-2223.

649 Herbomel, P., Thisse, B. and Thisse, C. (1999). Ontogeny and behaviour of early 650 macrophages in the zebrafish embryo. Development 126, 3735-3745.

--- (2001). Zebrafish early macrophages colonize cephalic mesenchyme and developing brain, retina, and epidermis through a M-CSF receptor-dependent invasive process. Dev Biol 238, 274-288.

Hess, I. and Boehm, T. (2012). Intravital imaging of thymopoiesis reveals dynamic lympho-epithelial interactions. Immunity 36, 298-309.

656 Kana, V., Desland, F. A., Casanova-Acebes, M., Ayata, P., Badimon, A., Nabel, 657 E., Yamamuro, K., Sneeboer, M., Tan, I. L., Flanigan, M. E., et al. (2019). CSF-1 658 controls cerebellar microglia and is required for motor function and social interaction. 659 J Exp Med.

660 Kuil, L. E., Oosterhof, N., Ferrero, G., Mikulasova, T., Hason, M., Dekker, J., 661 Rovira, M., van der Linde, H. C., van Strien, P. M., de Pater, E., et al. (2020). 
Zebrafish macrophage developmental arrest underlies depletion of microglia and reveals Csf1r-independent metaphocytes. Elife $\mathbf{9}$.

Kuil, L. E., Oosterhof, N., Geurts, S. N., van der Linde, H. C., Meijering, E. and van Ham, T. J. (2019). Reverse genetic screen reveals that II34 facilitates yolk sac macrophage distribution and seeding of the brain. Dis Model Mech 12.

Lareau, C., lyer, S., Langenau, D. M. and Aryee, M. (2017). Single Cell inDrops RNA-Seq Visualization of Adult Zebrafish Whole Kidney Marrow. Harvard University. Available at https://molpath.shinyapps.io/zebrafishblood/.

Li, Q. and Barres, B. A. (2018). Microglia and macrophages in brain homeostasis and disease. Nat Rev Immunol 18, 225-242.

Lin, H., Lee, E., Hestir, K., Leo, C., Huang, M., Bosch, E., Halenbeck, R., Wu, G., Zhou, A., Behrens, D., et al. (2008). Discovery of a cytokine and its receptor by functional screening of the extracellular proteome. Science 320, 807-811.

Mazzolini, J., Le Clerc, S., Morisse, G., Coulonges, C., Kuil, L. E., van Ham, T. J., Zagury, J. F. and Sieger, D. (2019). Gene expression profiling reveals a conserved microglia signature in larval zebrafish. Glia.

Murayama, E., Kissa, K., Zapata, A., Mordelet, E., Briolat, V., Lin, H. F., Handin, R. I. and Herbomel, P. (2006). Tracing hematopoietic precursor migration to successive hematopoietic organs during zebrafish development. Immunity 25, 963975.

Nimmerjahn, A., Kirchhoff, F. and Helmchen, F. (2005). Resting microglial cells are highly dynamic surveillants of brain parenchyma in vivo. Science 308, 1314-1318.

Oosterhof, N., Chang, I. J., Karimiani, E. G., Kuil, L. E., Jensen, D. M., Daza, R., Young, E., Astle, L., van der Linde, H. C., Shivaram, G. M., et al. (2019). Homozygous Mutations in CSF1R Cause a Pediatric-Onset Leukoencephalopathy and Can Result in Congenital Absence of Microglia. Am J Hum Genet.

Oosterhof, N., Kuil, L. E., van der Linde, H. C., Burm, S. M., Berdowski, W., van Ijcken, W. F. J., van Swieten, J. C., Hol, E. M., Verheijen, M. H. G. and van Ham, T. J. (2018). Colony-Stimulating Factor 1 Receptor (CSF1R) Regulates Microglia Density and Distribution, but Not Microglia Differentiation In Vivo. Cell Rep 24, 12031217 e1206. 
Paloneva, J., Manninen, T., Christman, G., Hovanes, K., Mandelin, J., Adolfsson, R., Bianchin, M., Bird, T., Miranda, R., Salmaggi, A., et al. (2002). Mutations in two genes encoding different subunits of a receptor signaling complex result in an identical disease phenotype. Am J Hum Genet 71, 656-662.

697 Paolicelli, R. C., Bolasco, G., Pagani, F., Maggi, L., Scianni, M., Panzanelli, P., 698 Giustetto, M., Ferreira, T. A., Guiducci, E., Dumas, L., et al. (2011). Synaptic 699 pruning by microglia is necessary for normal brain development. Science 333, 14567001458.

Parichy DM, R. D., Paw B, Zon LI, Johnson SL (2000). An orthologue of the kitrelated gene fms is required for development of neural crest-derived xanthophores and a subpopulation of adult melanocytes in the zebrafish, Danio rerio. Development 127, 3031-3044.

Peri, F. and Nusslein-Volhard, C. (2008). Live imaging of neuronal degradation by microglia reveals a role for v0-ATPase a1 in phagosomal fusion in vivo. Cell 133, 916927.

Prinz, M., Jung, S. and Priller, J. (2019). Microglia Biology: One Century of Evolving Concepts. Cell 179, 292-311.

Rademakers, R., Baker, M., Nicholson, A. M., Rutherford, N. J., Finch, N., SotoOrtolaza, A., Lash, J., Wider, C., Wojtas, A., DeJesus-Hernandez, M., et al. (2011). Mutations in the colony stimulating factor 1 receptor (CSF1R) gene cause hereditary diffuse leukoencephalopathy with spheroids. Nat Genet 44, 200-205.

Rojo, R., Raper, A., Ozdemir, D. D., Lefevre, L., Grabert, K., WollscheidLengeling, E., Bradford, B., Caruso, M., Gazova, I., Sanchez, A., et al. (2019). Deletion of a Csf1r enhancer selectively impacts CSF1R expression and development of tissue macrophage populations. Nat Commun 10, 3215.

Sasmono, R. T., Oceandy, D., Pollard, J. W., Tong, W., Pavli, P., Wainwright, B. J., Ostrowski, M. C., Himes, S. R. and Hume, D. A. (2003). A macrophage colonystimulating factor receptor-green fluorescent protein transgene is expressed throughout the mononuclear phagocyte system of the mouse. Blood 101, 1155-1163.

Scapigliati, G., Fausto, A. M. and Picchietti, S. (2018). Fish Lymphocytes: An Evolutionary Equivalent of Mammalian Innate-Like Lymphocytes? Front Immunol 9, 971. 
Sierra, A., Paolicelli, R. C. and Kettenmann, H. (2019). Cien Años de Microglía: Milestones in a Century of Microglial Research. Trends in Neurosciences.

727

728

729

730

731

732

733

734

Squarzoni, P., Oller, G., Hoeffel, G., Pont-Lezica, L., Rostaing, P., Low, D., Bessis, A., Ginhoux, F. and Garel, S. (2014). Microglia modulate wiring of the embryonic forebrain. Cell Rep 8, 1271-1279.

Stanley, E. R. and Chitu, V. (2014). CSF-1 receptor signaling in myeloid cells. Cold Spring Harb Perspect Biol 6.

Stanley, E. R. H., P.M. (1977). Factors Regulating Macrophage Production and Growth. Purification and some properties of the colony stimulating factor from medium conditioned by mouse L cells. J Biol Chem 252, 8.

Susaki, E. A., Tainaka, K., Perrin, D., Yukinaga, H., Kuno, A. and Ueda, H. R. (2015). Advanced CUBIC protocols for whole-brain and whole-body clearing and imaging. Nat Protoc 10, 1709-1727.

Thisse, C. and Thisse, B. (2008). High-resolution in situ hybridization to whole-mount zebrafish embryos. Nat Protoc 3, 59-69.

Tong, C. K. and Vidyadaran, S. (2016). Role of microglia in embryonic neurogenesis. Exp Biol Med (Maywood) 241, 1669-1675.

Wang, Y., Szretter, K. J., Vermi, W., Gilfillan, S., Rossini, C., Cella, M., Barrow, A. D., Diamond, M. S. and Colonna, M. (2012). IL-34 is a tissue-restricted ligand of CSF1R required for the development of Langerhans cells and microglia. Nat Immunol 13, 753-760.

Wittamer, V., Bertrand, J. Y., Gutschow, P. W. and Traver, D. (2011). Characterization of the mononuclear phagocyte system in zebrafish. Blood 117, 71267135.

Wu, S., Xue, R., Hassan, S., Nguyen, T. M. L., Wang, T., Pan, H., Xu, J., Liu, Q., Zhang, W. and Wen, Z. (2018). II34-Csf1r Pathway Regulates the Migration and Colonization of Microglial Precursors. Dev Cell 46, 552-563 e554.

Xu, J., Zhu, L., He, S., Wu, Y., Jin, W., Yu, T., Qu, J. Y. and Wen, Z. (2015). Temporal-Spatial Resolution Fate Mapping Reveals Distinct Origins for Embryonic and Adult Microglia in Zebrafish. Dev Cell 34, 632-641. 
755 Yu, T., Guo, W., Tian, Y., Xu, J., Chen, J., Li, L. and Wen, Z. (2017). Distinct 756 regulatory networks control the development of macrophages of different origins in 757 zebrafish. Blood 129, 509-519.

758 Zeisel, A. M.-M., A.B.; Codeluppi, S.; Lönnerberg P.; La Manno, G.; Juréus, A.; 759 Marques, S.; Munguba, H.; He, L.; Betsholtz, C.; Rolny, C.; Castelo-Branco, G.; 760 Hjerling-Leffler, J.; Linnarsson, S. (2015). Cell types in the mouse cortex and 761 hippocampus revealed by single-cell RNA-seq. Science 347, 5.

762 Zriwil, A., Boiers, C., Wittmann, L., Green, J. C., Woll, P. S., Jacobsen, S. E. and 763 Sitnicka, E. (2016). Macrophage colony-stimulating factor receptor marks and 764 regulates a fetal myeloid-primed B-cell progenitor in mice. Blood 128, 217-226. 
bioRxiv preprint doi: https://doi.org/10.1101/2020.11.04.368183; this version posted November 4, 2020. The copyright holder for this preprint (which was not certified by peer review) is the author/funder, who has granted bioRxiv a license to display the preprint in Figure $1 \quad$ perpetuity. It is made available under aCC-BY-NC-ND 4.0 International license.

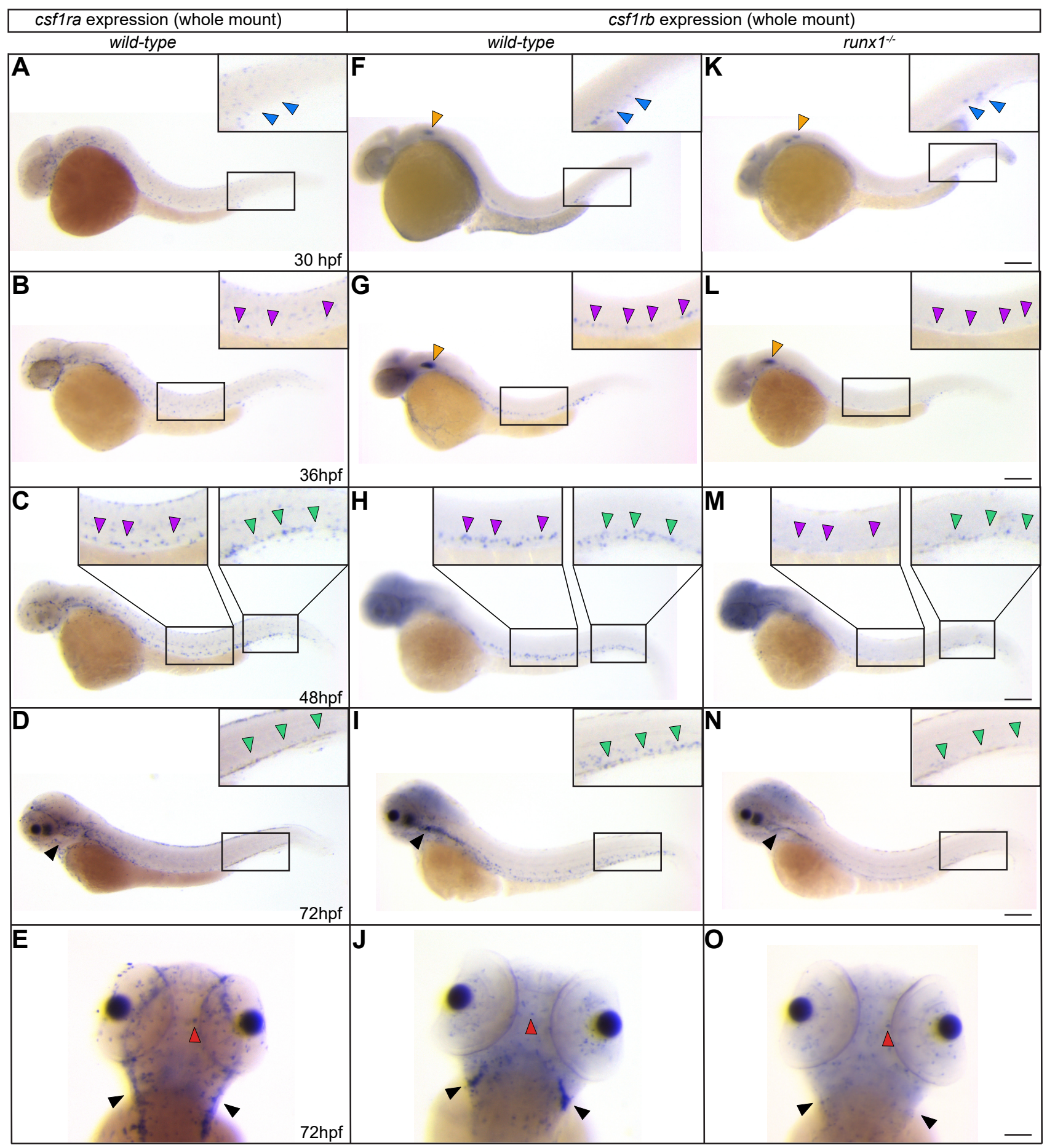

Figure 1. Csf1ra and csf1rb paralogs have nonoverlapping distribution during early development, except for microglia. Whole-mount in-situ hybridization (WISH) expression profiles of csf1ra (A-E) and csf1rb (F-J) in wild-type and csf1rb in runx1\% $(\mathrm{K}-\mathrm{O})$ embryos, at the indicated stages. All lateral views, except for $\mathrm{E}, \mathrm{J}$ and $\mathrm{O}$, shown in dorsal view. Orange and blue arrowheads indicate expression in the otic vesicle and posterior blood island $(\mathrm{PBI})$ region, respectively. Purple and green arrowheads indicate expression in the dorsal aorta (DA) and caudal hematopoietic tissue (CHT), respectively. Black arrowheads show bilateral thymi and red arrowheads are microglial cells. Scale bars: $200 \mu \mathrm{m}$ (30 and 36 hpf); $180 \mu \mathrm{m}$ (48 hpf); $150 \mu \mathrm{m}$ (72 hpf). 
Figure 2

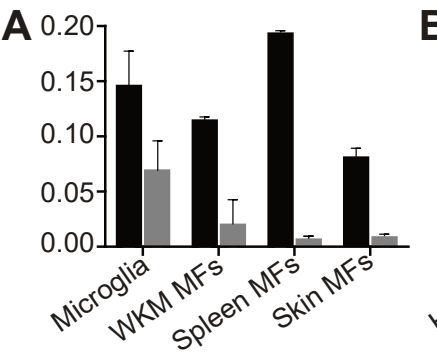

C

HSCs-thrombocytes

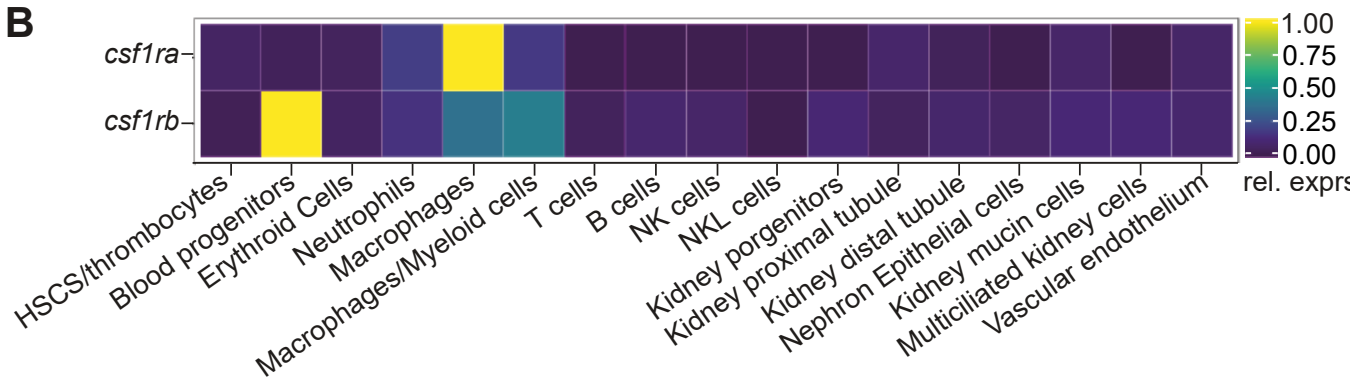

D csf1ra expression
$E$

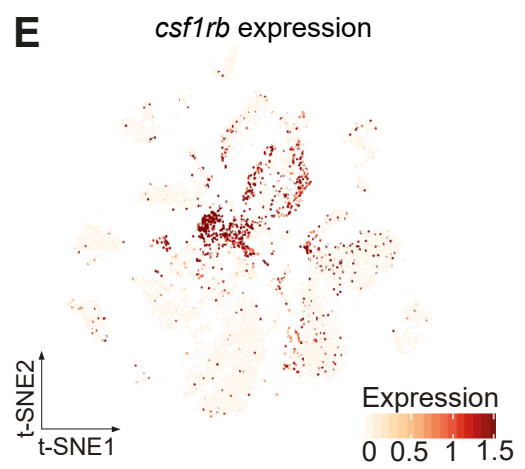

Figure 2. Characterization of csf1r paralog expression in adult hematopoietic cells. (A) QPCR expression for csf1ra and csf1rb in cd45:DsRed ; mhc2dab:GFP ${ }^{+}$mononuclear phagocytes sorted from adult zebrafish organs. Values on the $y$-axis indicate transcript expression normalized to ef1 $\alpha$ expression level. Error bars represent SEM ( $n=3)$. WKM: whole kidney marrow, MF: macrophages. (B-E) Expression profiles of csf1r paralogs in adult WKM hematopoietic and non-hematopoietic populations by single-cell RNAseq analysis, extracted from the public database from Laureau et al, 2017. Heatmap (B), 2D projection of the t-SNE analysis showing the distinct clusters identified in the adult WKM (C) and profiles of csf1ra (D) and csf1rb (E) across the clusters of the tSNE plot. Intensity of the color is proportional to the expression level. 
Figure 3
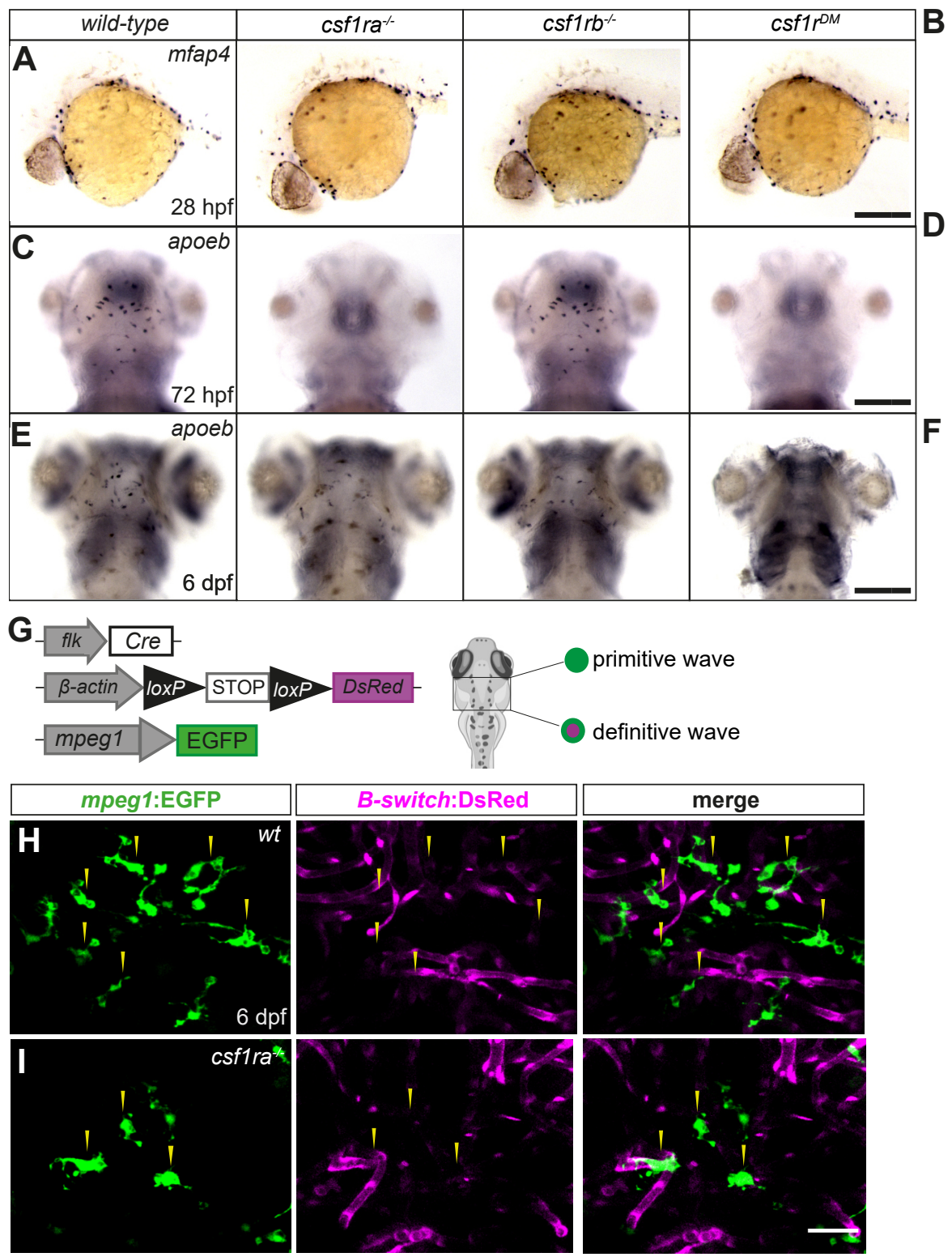

Figure 3. csf1ra and csf1rb are differently required for embryonic microglia development. $(\mathrm{A}, \mathrm{C}, \mathrm{E}) \mathrm{WISH}$ of the indicated genes in wild-type, csf1ra ${ }^{-/}, c s f 1 \mathrm{rb}^{-/}$and $c s f 1 \mathrm{r}^{\mathrm{DM}}$ siblings, at the stages indicated. All dorsal views except for $A$, shown in lateral view. Scale bars: $150 \mu \mathrm{m}(A, C)$; $100 \mu \mathrm{m}(\mathrm{E})$. (B, D, F) Quantification of $m f a p 4^{+}$primitive macrophages (B), apoeb $b^{+}$microglia at 3 $\mathrm{dpf}(\mathrm{D})$ and at $6 \mathrm{dpf}(\mathrm{F})$ in the indicated genotypes. Each symbol represents a single embryo/ larvae and error bars represent mean \pm SEM. Differences between groups were analyzed by Students t-test $\left[{ }^{* * *} p<0.001 ;{ }^{* * * *} p<0.0001\right]$. (G) Scheme of the transgenic lines used to discriminate the primitive and definitive microglia waves in 6 dpf larvae. $(H, I)$ Imaging by confocal microscopy of the optic tectum in $6 \mathrm{dpf}$ wild-type $(\mathrm{H})$ and csf1ra- ${ }^{-/}$(I) sibling larvae carrying the kdrl:Cre; ßactin:Switch-DsRed; mpeg1:EGFP triple transgene. GFP (left panels), DsRed (middle panels) and merge of both fluorescence channels (right panels) are shown. Images were taken with an inverted Zeiss LM780 confocal microscope using a $25 x$ waterimmersion objective. Scale bar: $50 \mu \mathrm{m}$. 

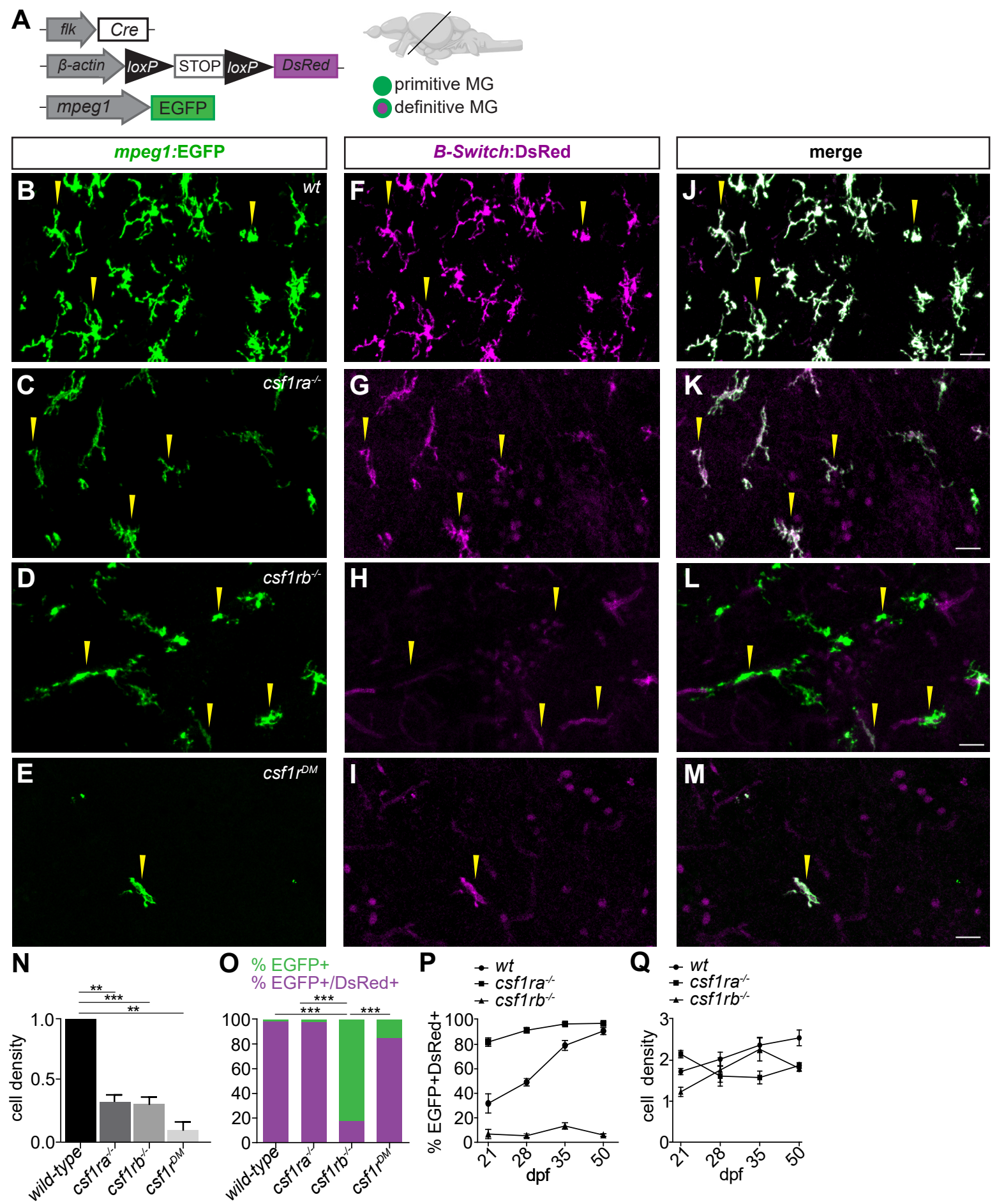

Figure 4. csf1rb is required for HSCs-derived microglia development. (A) Scheme of the transgenic lines used to discriminate primitive from definitive microglia in adult zebrafish brains.

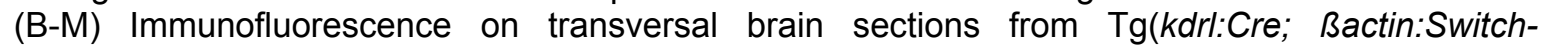
DsRed; mpeg1:EGFP) triple transgenic adult wild-type (B, F, J), csf1ra ${ }^{-/}(\mathrm{C}, \mathrm{G}, \mathrm{K}), \mathrm{csf1}_{\mathrm{rb}} \mathrm{r}^{-/}$(D, $\mathrm{H}, \mathrm{L})$ and $\operatorname{csf1r}^{D M}(\mathrm{E}, \mathrm{I}, \mathrm{M})$ fish. GFP (left panels), DsRed (middle panels) and merge of both fluorescence channels (right panels) are shown. $(\mathrm{N}, \mathrm{O})$ Quantification of microglia density (GFP ${ }^{+}$cells/100 $\left.\mu \mathrm{m} 2\right)(\mathrm{N})$ and percentage of $\mathrm{GFP}^{+}$DsRed- (green) embryonic versus GFP ${ }^{+}$DsRed+ (purple) adult microglia $(O)$ in each genotype. Bars represent the mean \pm SEM $(n=4)$. For each individual, cells were counted on ten, $30 \mu \mathrm{m}$-thick brain sections from rostral to caudal. Differences between groups were analyzed by One-way ANOVA test ${ }^{* * *} p<0.0005$.]. Images were taken with an inverted Zeiss LM780 confocal using a 20x objective. Scale bars $=50 \mu \mathrm{m}$. (P,Q) Percentage of $\mathrm{GFP}^{+}$DsRed $^{+}$adult microglia $(P)$ and microglia density (cells/1 mm3) (Q) at 21, 28, 35 and $50 \mathrm{dpf}$ for each genotype. Cells were counted on tissue-cleared whole brains as described in Ferrero et al, 2018. Cell counts were limited to the optic tectum and hindbrain areas. Between $4(21 \mathrm{dpf}, 28$ dpf) and 6 (50 dpf) images per brain were acquired, with an average z-stack of $400 \mu \mathrm{m}$., using a Zeiss LM780 confocal with a $25 x$ water-immersion objective. 
Figure 5
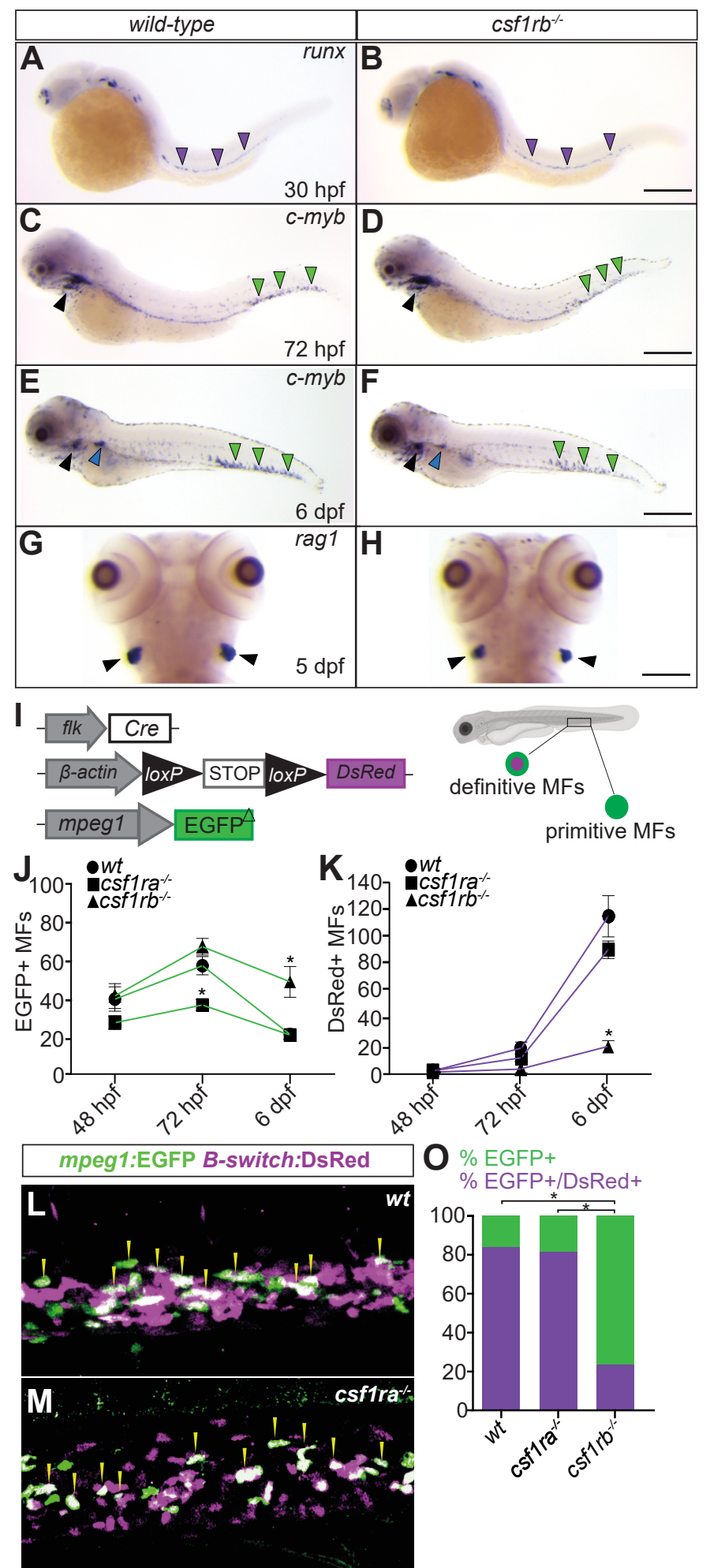

$\mathbf{O}$

\% EGFP+
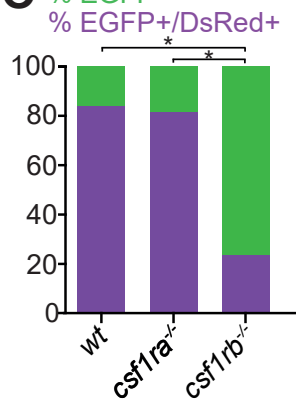

Figure 5. Loss of csf1rb function impairs the development of definitive macrophages. $(\mathrm{A}, \mathrm{H}) \mathrm{WISH}$ of the indicated genes in wild-type and csf1rb- siblings at the stages indicated. All lateral views except for $\mathrm{G}$ and $\mathrm{H}$, shown in dorsal. The purple arrowheads indicate run $\times 1^{+} \mathrm{HSCs}$ along the dorsal aorta. The green and blue arrowheads show $c m y b^{+}$hematopoietic progenitors in the $\mathrm{CHT}$ and in the pronephros, respectively. Black arrowheads indicate $\mathrm{rag}^{+}$or $\mathrm{cmyb}^{+}$lymphoid progenitors in the thymus. Scale bar: $200 \mu \mathrm{m}(\mathrm{A}, \mathrm{B}), 150$ $\mu \mathrm{m}(\mathrm{C}, \mathrm{D}, \mathrm{G}, \mathrm{H}), 100 \mu \mathrm{m}(\mathrm{E}, \mathrm{F})$. (I-O) Confocal imaging analysis of definitive myelopoiesis in csf1r mutant embryos and larvae (I) Scheme of the transgenic lines used to discriminate the primitive and definitive myelopoiesis waves in the CHT. $(\mathrm{J}, \mathrm{K})$ Quantification of $\mathrm{GFP}^{+}$DsRed ${ }^{-}$primitive $(\mathrm{J})$ and $\mathrm{GFP}^{+} \mathrm{DsRed}^{+}$definitive macrophages $(\mathrm{K})$ in wild-type, csf1 $\mathrm{ra}^{-/}$and $c s f 1 \mathrm{rb}^{-/}$ carrying the kdrl:Cre; Bactin:Switch-DsRed; mpeg1:EGFP triple transgene, at the indicated developmental stages $(n=4$; symbols represent mean \pm SEM). (L-N) Confocal imaging and quantification of the percentage $(\mathrm{O})$ of $\mathrm{GFP}^{+}$DsRed $^{-}$primitive macrophages (green) versus $\mathrm{GFP}^{+} \mathrm{DsRed}^{+}$definitive macrophages (purple) in the CHT of $6 \mathrm{dpf}$ wild-type and csf1r mutant larvae. Bars in the graph represent the mean of 4 larvae for each group. Cells in the $\mathrm{CHT}$ were quantified in four contiguous $385 \mu \mathrm{m} 2$ fields per $\mathrm{CHT}$, with an average $100 \mu \mathrm{m}$ z-stack, from caudal to rostral. Images were taken with an inverted Zeiss LM780 confocal microscope, using a $25 x$ water-immersion objective. Difference between groups were analyzed by Kruskal-Wallis test $\left[{ }^{*} P<0.05\right]$.

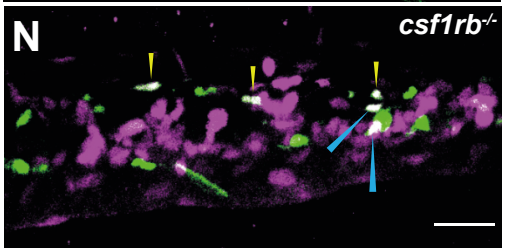


Figure 6
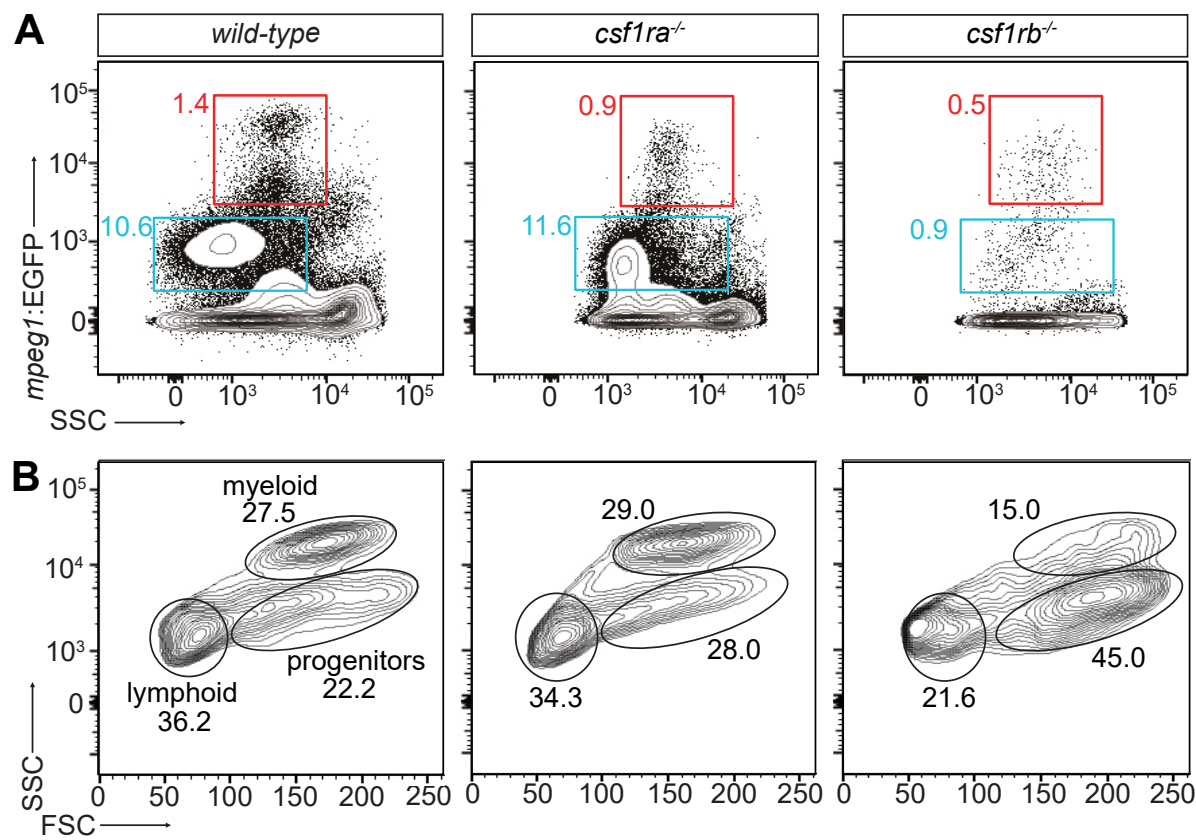

Figure 6. Adult zebrafish csf1rb mutant display hematopoietic deficiencies. (A, B) Flow cytometry analysis of WKM cell suspensions from wild-type, csf1 $\mathrm{ra}^{-/}$and $c s f 1 \mathrm{rb}^{-/}$adult fish carrying the mpeg1:EGFP reporter. (A) The mpeg1:EGFPhi fractions identify mature macrophages (red frames), while the mpeg1:EGFPlo fractions contain mainly IgM-expressing $B$ lymphocytes (blue frames). (B) Scatter profiles of WKM in typical wild-type (left panel), csf1 ra- (middle panel) and $c s f 1 \mathrm{rb}^{-/}$(right panel) adult fish. Number in plots indicate percent of cells in circled myeloid, progenitor and lymphoid gates. Means \pm SEM for 3 individuals are indicated in the text. 
bioRxiv preprint doi: https://doi.org/10.1101/2020.11.04.368183; this version posted November 4,2020 . The copyright holder for this preprint (which was not certified by peer review) is the author/funder, who has granted bioRxiv a license to display the preprint in perpetuity. It is made available under aCC-BY-NC-ND 4.0 International license.

Table 1. qPCR primers used throughout the paper

\begin{tabular}{|c|c|c|}
\hline Gene & Forward Primer & Reverse Primer \\
\hline ef1 $\alpha$ & GAGAAGTTCGAGAAGGAAGC & CGTAGTATTTGTGGTCTCG \\
\hline$m p e g 1.1$ & CCCACCAAGTGAAAGAGG & GTGTTTATTGTTTTCAATGG \\
\hline$c s f 1 r a$ & ATGACCATACCCAACTTCC & AGTTTGTTGGTCTGGATGTG \\
\hline$c s f 1 r b$ & TCGGTCTTGCTAGAGACATC & ATGACCAGACATCACTTTGG \\
\hline
\end{tabular}




\section{Figure S1}

A

genomic DNA

processed transcript

\begin{tabular}{|l|l|l|}
\hline exon 11 & $\begin{array}{c}\text { intron } 11 \\
(86 \mathrm{bp})\end{array}$ \\
\hline
\end{tabular}

\begin{tabular}{|l|l|}
\multicolumn{1}{c}{$\downarrow$} & $\square$ \\
\hline exon 11 & exon 12 \\
\hline
\end{tabular}

Csf1rb

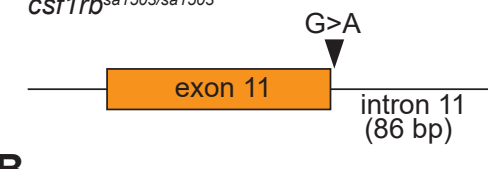

B
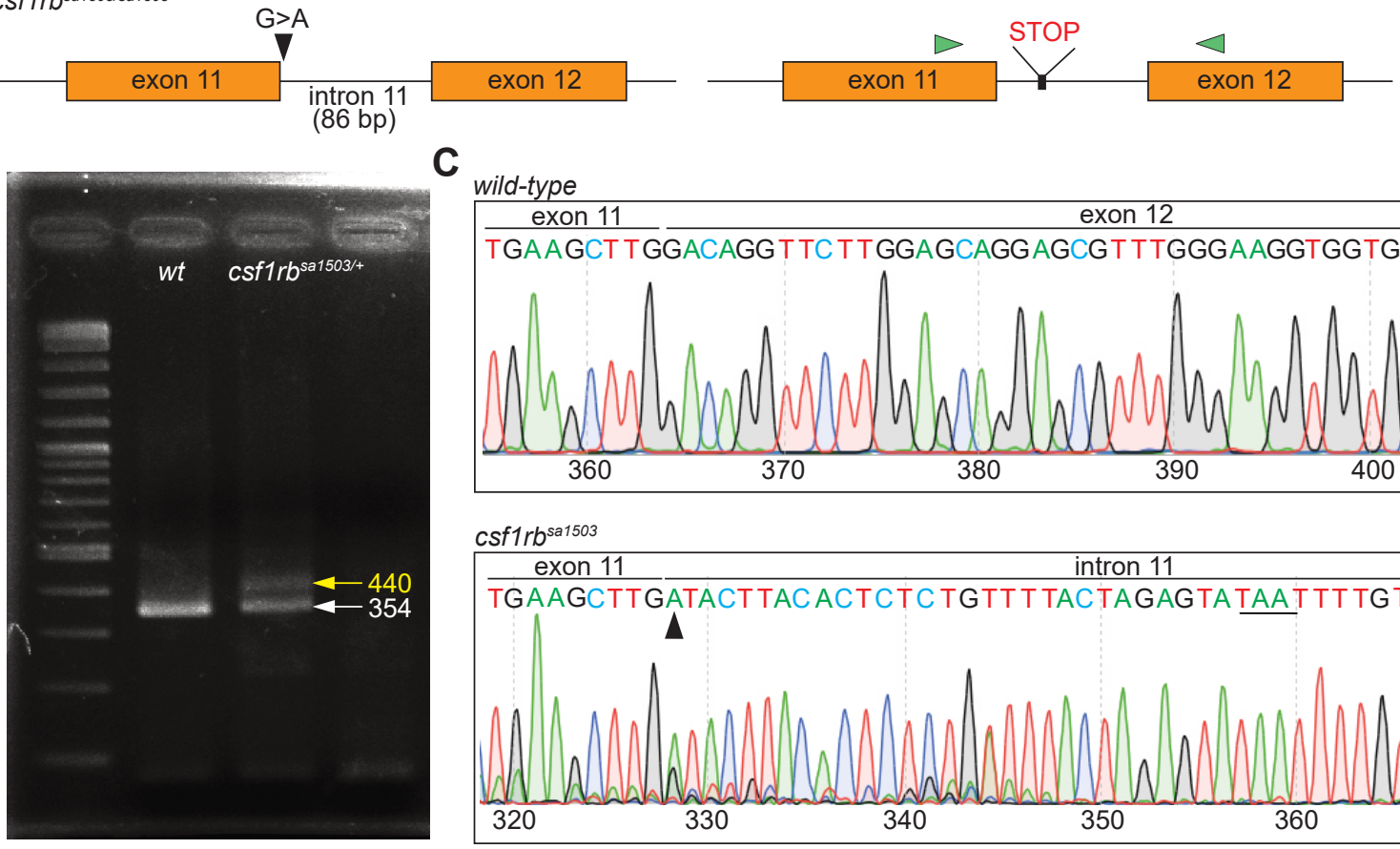

C
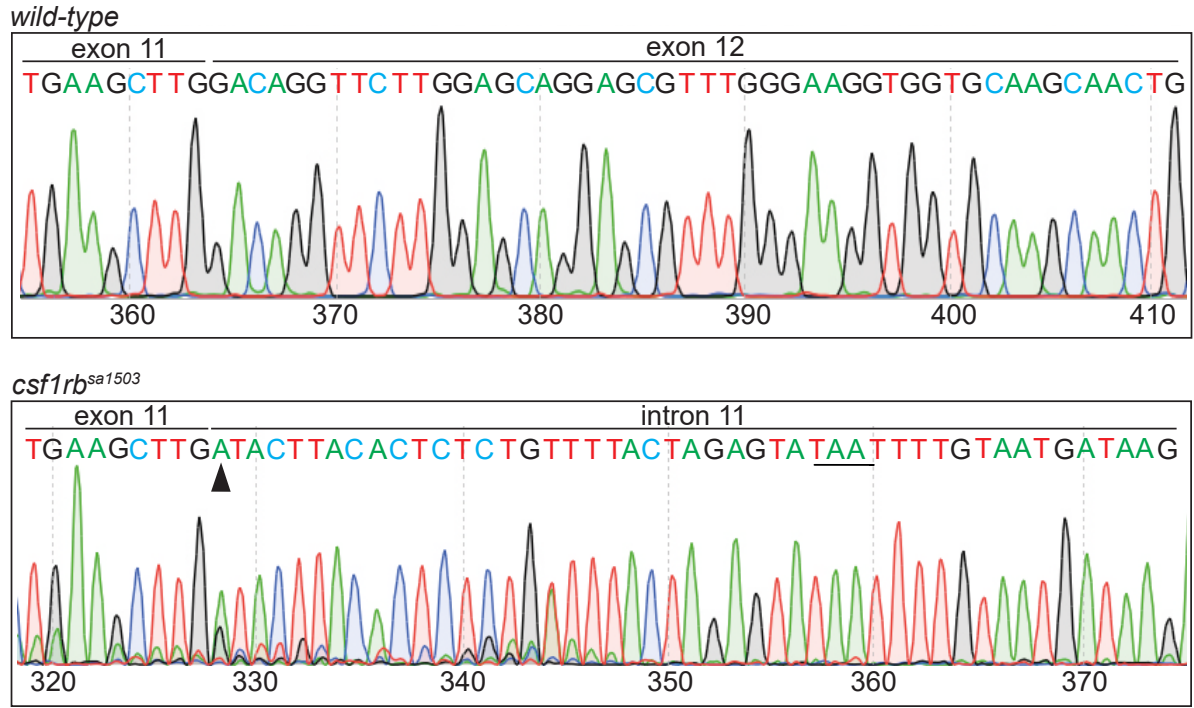

Figure S1. Characterization of the csf1rbsa1503 mutant line. (A) Schematic view of the exons 11 and 12 of the csf1rb gene and the alteration caused by the splice mutation (black arrowhead) in the csf1rb ${ }^{\text {sa1503 line, }}$ which leads to the inclusion of 86-bp from intron 11 and the introduction of a premature stop codon in the coding sequence. Green arrowheads indicate the position of the primers used for PCR amplification (B) RTPCR on whole brain isolated from adult wild-type and csf1rb $b^{\text {sa1503/+ }}$ mutant, showing the spliced wild-type

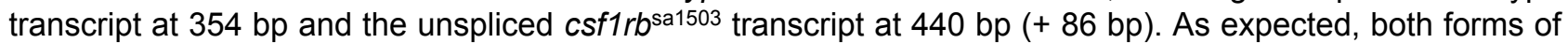
the transcript are found in the heterozygous mutant. (C) Sequence chromatograms show the G>A substitution (black arrowhead) and the premature stop codon TAA (underlined) in the $c s f 1 r b^{\text {sa1503 }}$ mutant. 
bioRxiv preprint doi: https://doi.org/10.1101/2020.11.04.368183; this version posted November 4,2020 . The copyright holder for this

preprint (which was not certified by peer review) is the author/funder, who has granted bioRxiv a license to display the preprint in perpetuity. It is made available under aCC-BY-NC-ND 4.0 International license.

Figure S2

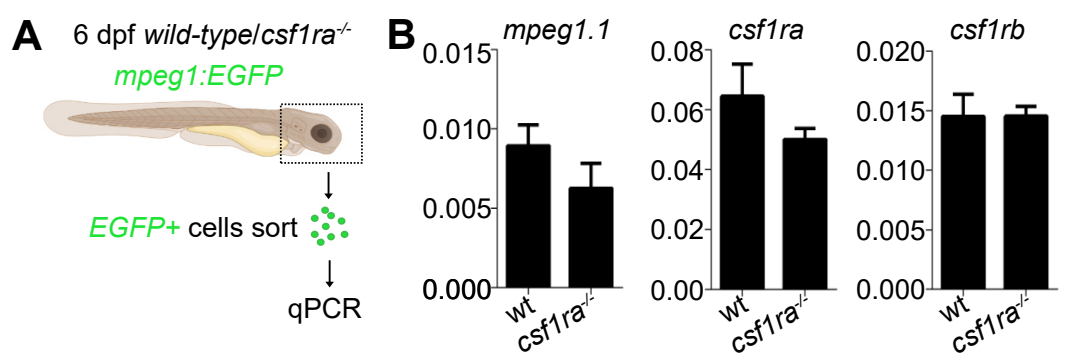

Figure S2. Comparison of csf1rb expression in 6 dpf wild-type and csf1 ra- head macrophages. (A) Experimental outline. (B) qPCR analysis of gene expression for mpeg1.1, csf1ra and csf1rb in sorted mpeg1:EGFP ${ }^{+}$cells. Error bars represent SEM $(n=3)$. Values on the $y$-axis indicate transcript expression normalized to ef1 $\alpha$ expression level. 